\title{
The impact of large-scale tides on cosmological distortions via redshift-space power spectrum
}

\author{
Kazuyuki Akitsu ${ }^{1,2}$ and Masahiro Takada ${ }^{1}$ \\ ${ }^{1}$ Kavli Institute for the Physics and Mathematics of the Universe (WPI), \\ The University of Tokyo Institutes for Advanced Study (UTIAS), \\ The University of Tokyo, Chiba 277-8583, Japan \\ ${ }^{2}$ Department of Physics, Graduate School of Science, \\ The University of Tokyo, 7-3-1 Hongo, Bunkyo-ku, Tokyo 113-0033, Japan
}

(Dated: November 2, 2017)

\begin{abstract}
Although the large-scale perturbations beyond a finite-volume survey region are not direct observables, these affect measurements of clustering statistics of small-scale (sub-survey) perturbations in the large-scale structure, compared with the ensemble average, via the mode-coupling effect. In this paper we show that the large-scale tides induced by scalar perturbations cause apparent anisotropic distortions in the redshift-space power spectrum of galaxies in a way depending on an alignment between the tides, the wavevector of small-scale modes and the line-of-sight direction. Using the perturbation theory of structure formation, we derive the response function of the redshift-space power spectrum to the large-scale tides. We then investigate the impact of the large-scale tides on estimation of cosmological distances and the redshift-space distortion parameter via the measured redshift-space power spectrum for a hypothetical large-volume survey, based on the Fisher matrix formalism. To do this, we treat the large-scale tides as a signal, rather than an additional source of the statistical errors, and show that a degradation in the parameter is restored if we can employ the prior on the rms amplitude expected for the standard cold dark matter (CDM) model. We also discuss whether the large-scale tides can be constrained at an accuracy better than the CDM prediction, if the effects up to a larger wavenumber in the nonlinear regime can be included.
\end{abstract}

\section{INTRODUCTION}

A number of wide-area and deep galaxy surveys are ongoing and planned, aimed at revealing the nature of primordial perturbations, the physics in the early universe, the curvature of the universe, the origin of the cosmic acceleration as well as weighing the neutrino mass via a measurement of large-scale structure probes such as weak gravitational lensing, baryon acoustic oscillations (BAO), galaxy clustering and redshift-space distortions [e.g., 1 1-3]. In particular, when combined with the high-precision measurement of cosmic microwave background (CMB) anisotropies, large-scale structure probes allow one to study the time evolution of the perturbations over cosmic ages, which is sensitive to the aforementioned physics and cosmological parameters [e.g. 4.

The linear perturbation theory can accurately describe the time evolution of large-scale perturbations in structure formation, based on the standard $\Lambda$ and cold dark matter dominated cosmology with Gaussian adiabatic initial conditions (hereafter $\Lambda \mathrm{CDM}$ ) [5], which successfully reproduces the high-precision measurements of CMB anisotropies, yielding stringent constraints on the cosmological parameters [6. The linear theory, however, breaks down in the latetime universe, which is relevant for galaxy surveys, because the nonlinear structure formation induces a mode coupling between different Fourier modes of the perturbations owing to the nature of nonlinear, long-range gravity [7, 8, for a thorough review]. As a result, the power spectrum of large-scale structure probes, measured from a galaxy survey, no longer carries the full information unlike the CMB anisotropies, and the statistical properties display a substantial non-Gaussianity that is described by higher-order correlation functions 9 . A better understanding of the nonlinear structure formation is thus required in order to attain the full potential of wide-area galaxy surveys.

Even though wide-area galaxy surveys are to cover a huge cosmological volume, there is an unavoidable uncertainty in the statistical analysis of large-scale structure probes arising due to finiteness of the survey volume as well as the nonlinear mode coupling, as studied in Refs. [10 31. A finite-volume survey realization is generally embedded into large-scale perturbations that are not directly observable - which we hereafter call "super-survey modes" [10, 25]. Although the super-survey modes have small amplitudes and are well in the linear regime for a wide-area galaxy survey, it causes a non-negligible effect on the small-scale perturbations due to the nonlinear mode coupling, compared with the statistical accuracies in measurements of the small-scale perturbations. Hence, it is necessary to include the effects in the cosmological analysis [e.g. 32] in order not to have an biased estimation in cosmological parameters as well as not to have too optimistic cosmological constraints.

The physical effects of super-survey modes on structure formation at equal time arise from the second-derivative tensor of the large-scale gravitational field due to the equivalence principle [16, 30. The tensor is decomposed into two modes: the trace part or the large-scale density contrast and the trace-less tensor which we hereafter call the large-scale tides. While the effect of the large-scale density contrast is well studied in previous studies [e.g. 10, the 
effect of the large-scale tides has not been fully studied, except for some studies [26, 29, 31, 33. In our previous study [30, we showed that the large-scale tides cause an anisotropic clustering in the redshift-space power spectrum. The effect mimics the redshift-space distortion effect [34, 35] arising from the peculiar velocities of large-scale structure tracers as well as the Alcock-Paczynski (AP) distortion 36 38] arising from the use of an incorrect cosmological model in the clustering analysis. Yet we studied only the partial effect, the effect of the large-scale tides on the real-space clustering; in other words, we did not include the effect of the large-scale tides on the redshift-space distortion as well as a modulation in the mapping between real- and redshift-space distributions of galaxies.

Hence, the purpose of this paper is to study the effects of the large-scale tides on the redshift-space power spectrum, based on the perturbation theory [7. To do this, we derive the response functions of the redshift-space power spectrum to super-survey modes, which describe how the super-survey modes for a given survey realization affect the redshiftspace power spectrum as a function of wavevector $\mathbf{k}$ and the line-of-sight direction, say $\hat{\mathbf{n}}$, relative to the large-scale tides. We then discuss the impact of the tides on estimation of cosmological distances and the redshift-space distortion parameter via a measurement of the redshift-space power spectrum for a hypothetical large-volume galaxy survey, using the Fisher matrix formalism.

The rest of this paper is organized as follows. In $\$$ II], we define the super-survey modes and introduce its isotropic component and anisotropic components. In \$III, we derive the response function of the redshift-space power spectrum to super-survey modes by considering the squeezed-limit bispectrum that is a cross-correlation of the super-survey modes with the redshift-space power spectrum estimator. In \$IV we study the impact of the super-survey modes on cosmological parameter estimation from a measurement of the redshift-space power spectrum, including the AP test, based on the Fisher information matrix analysis. \$V is devoted to the discussion. In Appendix A we give expressions for the multipole expansion of the redshift-space power spectrum in the presence of the super-survey modes.

\section{PRELIMINARIES}

The redshift-space density field of galaxies observed in a finite-volume survey region can be expressed, using the survey window function $W(\mathbf{x})$, following the formulation in Ref. [10]:

$$
\delta_{s W}(\mathbf{x})=W(\mathbf{x}) \delta_{s}(\mathbf{x})
$$

where $\delta_{s W}(\mathbf{x})$ is the observed density field, $\delta_{s}(\mathbf{x})$ is the underlying true density field in redshift space, and the survey window is defined in that $W(\mathbf{x})=1$ if $\mathbf{x}$ is inside the survey geometry, and otherwise $W(\mathbf{x})=0$. Throughout this paper we assume that a survey window is given in the background comoving coordinate. The survey volume is defined in terms of the survey window as

$$
V_{W}=\int \mathrm{d}^{3} \mathbf{x} W(\mathbf{x})
$$

In the following we assume a well-behaved survey window for simplicity; we do not consider effects of masks that might cause additional mode-coupling between high- $k$ modes in the observed power spectrum. The Fourier transform of the density field is

$$
\tilde{\delta}_{s W}(\mathbf{k})=\int \frac{\mathrm{d}^{3} \mathbf{q}}{(2 \pi)^{3}} \tilde{W}(\mathbf{q}) \tilde{\delta}_{s}(\mathbf{k}-\mathbf{q})
$$

where quantities with tilde symble such as $\tilde{\delta}_{s}(\mathbf{k})$ are their Fourier transforms. The survey window $\tilde{W}(\mathbf{k})$ is nonvanishing for $k \ll 1 / L$, while $\tilde{W}(\mathbf{k}) \simeq 0$ for $k \gg 1 / L$, where $L$ is a typical scale of the survey volume. The above equation explicitly shows that the Fourier transform of the observed field has a contribution from long-wavelength modes beyond a survey window, i.e. super-survey modes, via a convolution with the survey window.

Redshift-space distortion (RSD) effect due to peculiar velocities of galaxies causes a modulation in the observed clustering pattern of galaxies along the line-of-sight direction. Thus the RSD effect violates a statistical isotropy of the galaxy distribution. For this reason the monopole power spectrum, measured by the azimuthal-angle average of power spectrum over $\mathrm{d} \Omega_{\mathbf{k}}$, cannot carry the full information. Instead a standard approach to quantify the redshift-space clustering of galaxies is using the power spectrum, given as a function of the three-dimensional wavevector k. An estimator of the power spectrum for a given survey window is defined as

$$
\hat{P}_{s}(\mathbf{k}) \equiv \frac{1}{V_{W}} \int_{\mathbf{k}^{\prime} \in \mathbf{k}} \frac{\mathrm{d}^{3} \mathbf{k}^{\prime}}{V_{\mathbf{k}}} \tilde{\delta}_{s W}(\mathbf{k}) \tilde{\delta}_{s W}(-\mathbf{k})
$$


where the integration is done over a volume element around the mode $\mathbf{k}$ (a target wavevector for the power spectrum measurement), and $V_{\mathbf{k}}$ is the volume: $V_{\mathbf{k}} \equiv \int_{\mathbf{k}^{\prime} \in \mathbf{k}} \mathrm{d}^{3} \mathbf{k}^{\prime}$. If a bin width around the bin $\mathbf{k}$ is given by $\Delta k, V_{\mathbf{k}} \simeq(\Delta k)^{3}$. This definition does not include an angle average of $\mathrm{d} \Omega_{\mathbf{k}}$, unlike a definition of the monopole power spectrum. Hence, at this point, the redshift-space power spectrum $\hat{P}_{s}(\mathbf{k})$ is given as a function of the three-dimensional vector, $\mathbf{k}$. A standard method usually further assumes the statistical isotropy in the two-dimensional plane (angular direction) perpendicular to the line-of-sight direction, and then uses the power spectrum given as a function of two-dimensional vector $\left(k_{\|}, k_{\perp}\right)$, where $k_{\|}$is the line-of-sight direction component of $\mathbf{k}, \mathbf{k}_{\perp}$ is the vector in the two-dimensional plane perpendicular to the line-of-sight direction, and $k_{\perp}=\left|\mathbf{k}_{\perp}\right|$. Here we do not introduce the angle average over $\mathrm{d} \varphi_{\mathbf{k}_{\perp}}$ in the perpendicular plane, where $\varphi_{\mathbf{k}_{\perp}}$ is defined via $\mathbf{k}_{\perp}=k_{\perp}\left(\cos \varphi_{\mathbf{k}}, \sin \varphi_{\mathbf{k}}\right)$, and keep the general definition of $\hat{P}_{s}(\mathbf{k})$ because the large-scale tides generally cause anisotropic distortions in the redshift-space clustering pattern of galaxies in all three-dimensional directions [also see Ref. [39, for the similar discussion].

Given the definition of the redshift-space power spectrum,

$$
\left\langle\tilde{\delta}_{s}(\mathbf{k}) \tilde{\delta}_{s}\left(\mathbf{k}^{\prime}\right)\right\rangle \equiv(2 \pi)^{3} P_{s}(\mathbf{k}) \delta_{D}^{3}\left(\mathbf{k}+\mathbf{k}^{\prime}\right),
$$

where $\delta_{D}^{3}(\mathbf{k})$ is the Dirac delta function, the ensemble average of the estimator (Eq. 4 is found to be an unbiased estimator of the underlying power spectrum for modes with $k \gg 1 / L$ :

$$
\begin{aligned}
\left\langle\hat{P}_{s}(\mathbf{k})\right\rangle & =\frac{1}{V_{W}} \int_{\mathbf{k} \in \mathbf{k}^{\prime}} \frac{\mathrm{d}^{3} \mathbf{k}^{\prime}}{V_{\mathbf{k}}} \int \frac{\mathrm{d}^{3} \mathbf{q}}{(2 \pi)^{3}}|\tilde{W}(\mathbf{q})|^{2} P_{s}(\mathbf{k}-\mathbf{q}) \\
& \simeq \frac{1}{V_{W}} \int_{\mathbf{k} \in \mathbf{k}^{\prime}} \frac{\mathrm{d}^{3} \mathbf{k}^{\prime}}{V_{\mathbf{k}}} P_{s}(\mathbf{k}) \int \frac{\mathrm{d}^{3} \mathbf{q}}{(2 \pi)^{3}}|\tilde{W}(\mathbf{q})|^{2} \\
& \simeq \frac{P_{s}(\mathbf{k})}{V_{W}} \int_{\mathbf{k} \in \mathbf{k}^{\prime}} \frac{\mathrm{d}^{3} \mathbf{k}^{\prime}}{V_{\mathbf{k}}} \int \frac{\mathrm{d}^{3} \mathbf{q}}{(2 \pi)^{3}}|\tilde{W}(\mathbf{q})|^{2}=P_{s}(\mathbf{k}) .
\end{aligned}
$$

Here we used $P_{s}(\mathbf{k}-\mathbf{q}) \simeq P_{s}(\mathbf{k})$ over the integration rage of $\mathrm{d}^{3} \mathbf{q}$ which the window function supports and assumed that $P_{s}(\mathbf{k})$ is not a rapidly varying function within the $\mathbf{k}$-bin. In addition, we used the general identity for the window function [10]:

$$
V_{W}=\int \mathrm{d}^{3} \mathbf{x} W(\mathbf{x})^{n}=\int\left[\prod_{a=1}^{n} \frac{\mathrm{d}^{3} \mathbf{q}_{a}}{(2 \pi)^{3}} \tilde{W}\left(\mathbf{q}_{a}\right)\right](2 \pi)^{3} \delta_{D}^{3}\left(\mathbf{q}_{1 \ldots n}\right),
$$

where $\mathbf{q}_{1 \ldots n} \equiv \mathbf{q}_{1}+\mathbf{q}_{2}+\cdots+\mathbf{q}_{n}$.

Similarly to Takada \& Hu [10] and Akitsu et al. [30, we study effects of super-survey modes on the redshift-space power spectrum. The super-survey modes we focus on are the large-scale density contrast and the large-scale tides, defined in terms of the linear matter density fluctuation field as

$$
\begin{aligned}
\delta_{\mathrm{b}} & \equiv \frac{1}{V_{W}} \int \mathrm{d}^{3} \mathbf{x} W(\mathbf{x}) \tilde{\delta}_{\mathrm{m} L}(\mathbf{x})=\frac{1}{V_{W}} \int \frac{\mathrm{d}^{3} \mathbf{q}}{(2 \pi)^{3}} \tilde{\delta}_{\mathrm{m} L}(\mathbf{q}) \tilde{W}(-\mathbf{q}), \\
\tau_{i j} & \equiv \frac{1}{4 \pi G \bar{\rho}_{\mathrm{m}} a^{2} V_{W}} \int \mathrm{d}^{3} \mathbf{x} W(\mathbf{x})\left[\Phi_{, i j}(\mathbf{x})-\frac{\delta_{i j}^{K}}{3} \nabla^{2} \Phi(\mathbf{x})\right]=\frac{1}{V_{W}} \int \frac{\mathrm{d}^{3} \mathbf{q}}{(2 \pi)^{3}}\left(\hat{q}_{i} \hat{q}_{j}-\frac{\delta_{i j}^{K}}{3}\right) \tilde{\delta}_{\mathrm{m} L}(\mathbf{q}) \tilde{W}(-\mathbf{q}),
\end{aligned}
$$

where $\hat{q}_{i} \equiv q_{i} / q, \hat{q}_{i} \hat{q}^{i}=1, \delta_{i j}^{K}$ is the Kronecker delta, and $\Phi(\mathbf{x})$ is the gravitational potential field. Here we assumed that a survey volume is sufficiently large, and therefore the matter density field contributing the super-survey modes are in the linear regime, denoted as $\delta_{\mathrm{m} L}(\mathbf{x})$. Under this setting, $\left|\delta_{\mathrm{b}}\right|,\left|\tau_{i j}\right| \ll 1$. These super-survey modes are not direct observables and vary with survey realizations. For a particular survey realization, $\delta_{\mathrm{b}}, \tau_{i j}$ have particular constant values. The expectation values of the ensemble averages, i.e. the averages over different, possible survey realizations for a fixed volume, are computed if the linear matter power spectrum at long wavelengths for super-survey modes, $P^{L}(k)$, is given for a given cosmological model: $\left\langle\delta_{\mathrm{b}}\right\rangle=\left\langle\tau_{i j}\right\rangle=0$, and the variances are

$$
\begin{aligned}
& \sigma_{b}^{2} \equiv\left\langle\delta_{\mathrm{b}}^{2}\right\rangle=\frac{1}{V_{W}^{2}} \int \frac{d^{3} \mathbf{q}}{(2 \pi)^{3}} P^{L}(q)|W(\mathbf{q})|^{2} \\
& \left\langle\delta_{\mathrm{b}} \tau_{i j}\right\rangle=\frac{1}{V_{W}^{2}} \int \frac{d^{3} \mathbf{q}}{(2 \pi)^{3}}\left(\hat{q}_{i} \hat{q}_{j}-\frac{1}{3} \delta_{i j}^{K}\right) P^{L}(q)|W(\mathbf{q})|^{2}, \\
& \left\langle\tau_{i j} \tau_{l m}\right\rangle=\frac{1}{V_{W}^{2}} \int \frac{d^{3} \mathbf{q}}{(2 \pi)^{3}}\left(\hat{q}_{i} \hat{q}_{j}-\frac{1}{3} \delta_{i j}^{K}\right)\left(\hat{q}_{l} \hat{q}_{m}-\frac{1}{3} \delta_{l m}^{K}\right) P^{L}(q)|W(\mathbf{q})|^{2},
\end{aligned}
$$


where $P^{L}(q)$ is the linear matter power spectrum.

In this paper we consider an isotropic window for simplicity; $\tilde{W}(\mathbf{q})=\tilde{W}(q)$. In this case the variances of large-scale tides are simplified as

$$
\begin{aligned}
& \left\langle\delta_{\mathrm{b}} \tau_{i j}\right\rangle=0 \\
& \sigma_{\tau}^{2} \equiv\left\langle\left(\tau_{11}\right)^{2}\right\rangle=\left\langle\left(\tau_{22}\right)^{2}\right\rangle=\left\langle\left(\tau_{33}\right)^{2}\right\rangle=\frac{3}{4}\left\langle\left(\tau_{i j}\right)^{2}\right\rangle_{i \neq j}=\frac{4}{45 V_{W}^{2}} \int \frac{q^{2} \mathrm{~d} q}{2 \pi^{2}} P^{L}(q)|\tilde{W}(q)|^{2}=\frac{4}{45} \sigma_{\mathrm{b}}^{2} .
\end{aligned}
$$

\section{RESPONSES OF REDSHIFT-SPACE POWER SPECTRUM TO SUPER-SURVEY MODES}

\section{A. Redshift-space distortion effects}

In a redshift galaxy survey, the radial position of each galaxy needs to be inferred from its observed redshift. Here the observed redshift can be modified by a peculiar velocity of the galaxy through the Doppler effect, causing an apparent displacement of the inferred galaxy position from the true position:

$$
\mathbf{s}=\mathbf{x}+\frac{v_{\|}(\mathbf{x})}{\mathcal{H}(z)} \hat{\mathbf{n}}
$$

where $\mathbf{s}$ is the inferred position of a galaxy in redshift space, $\mathbf{x}$ is the true position in real space, $v_{\|}$is the radial component of the peculiar velocity, $\mathcal{H}(z)$ is the comoving Hubble rate, and $\hat{\mathbf{n}}$ is the unit vector of the line-of-sight direction. With this coordinate transformation, the density field in redshift space can be expressed as

$$
\rho_{s}(\mathbf{s})=\int \mathrm{d}^{3} \mathbf{x} \rho(\mathbf{x}) \delta_{D}^{3}\left(\mathbf{s}-\mathbf{x}-\frac{v_{\|}(\mathbf{x})}{\mathcal{H}(z)} \hat{\mathbf{n}}\right)
$$

where $\rho_{s}(\mathbf{s})$ or $\rho(\mathbf{x})$ denotes the redshift- or real-space density field of galaxies, respectively. In the following quantities with subscript " $s$ " denote their redshift-space quantities. Fourier-transforming Eq. 12 , $\int \mathrm{d}^{3} \mathbf{s} e^{i \mathbf{k} \cdot \mathbf{s}}$, yields

$$
\delta_{D}^{3}(\mathbf{k})+\tilde{\delta}_{s}(\mathbf{k})=\int \mathrm{d}^{3} \mathbf{x}[1+\delta(\mathbf{x})] e^{-i \mathbf{k} \cdot \mathbf{x}-i(\mathbf{k} \cdot \hat{\mathbf{n}}) \frac{v \|}{\mathcal{H}}}
$$

This transformation is exact even if multiple galaxies are mapped to the same position in redshift space, which can happen, e.g. in a nonlinear high-density region. Such multi-streaming regions are beyond the scope of this paper, and we ignore the effects in this paper for simplicity. In this setting we can rewrite Eq. (13) as

$$
\begin{aligned}
\tilde{\delta}_{s}(\mathbf{k}) & =\int \mathrm{d}^{3} \mathbf{x}\left[1+\delta(\mathbf{x})-\left|\frac{\partial s_{i}}{\partial x_{j}}\right|\right] e^{-i \mathbf{k} \cdot \mathbf{x}-i(\mathbf{k} \cdot \hat{\mathbf{n}}) \frac{v_{\|}}{\mathcal{H}}} \\
& \simeq \int \mathrm{d}^{3} \mathbf{x}\left[\delta(\mathbf{x})-\frac{1}{\mathcal{H}(z)} \frac{\partial v_{\|}}{\partial \hat{\mathbf{n}}} \cdot \hat{\mathbf{n}}\right] e^{-i \mathbf{k} \cdot \mathbf{x}-i(\mathbf{k} \cdot \hat{\mathbf{n}}) \frac{v_{\|}}{\mathcal{H}}}
\end{aligned}
$$

where we kept the peculiar velocity up to the linear order in an expansion of the Jacobian, $\left|\partial s_{i} / \partial x_{j}\right|$, assuming $\left|v_{\|} / \mathcal{H}\right| \ll 1$.

Using the perturbation theory of structure formation [7], we can express the redshift-space density field $\tilde{\delta}_{s}(\mathbf{k})$ in terms of the linear matter density field $\tilde{\delta}_{\mathrm{m} L}(\mathbf{k})$ as

$$
\tilde{\delta}_{s}(\mathbf{k} ; t) \equiv \sum_{n=1}^{\infty} \int\left[\prod_{a=1}^{\infty} \frac{\mathrm{d}^{3} \mathbf{k}_{a}}{(2 \pi)^{3}}\right] Z_{i}\left(\mathbf{k}_{1}, \ldots, \mathbf{k}_{i}\right) \tilde{\delta}_{\mathrm{m} L}\left(\mathbf{k}_{1}, t\right) \cdots \tilde{\delta}_{\mathrm{m} L}\left(\mathbf{k}_{i}, t\right)(2 \pi)^{3} \delta_{D}^{3}\left(\mathbf{k}_{1 \ldots i}-\mathbf{k}\right)
$$

where we have introduced the notation, $\mathbf{k}_{12 \ldots i} \equiv \mathbf{k}_{1}+\mathbf{k}_{2}+\cdots+\mathbf{k}_{i}$, and $Z_{i}\left(\mathbf{k}_{1}, \ldots, \mathbf{k}_{i}\right)$ is the mode-coupling kernel between different Fourier modes with $\mathbf{k}_{1}, \ldots, \mathbf{k}_{i}$. We throughout this paper employ a distant observer approximation for simplicity. In the following discussion we use the density fields up to the second-order, which are given as

$$
\begin{aligned}
\tilde{\delta}_{s}(\mathbf{k}) & \simeq \tilde{\delta}_{s}^{(1)}(\mathbf{k})+\tilde{\delta}_{s}^{(2)}(\mathbf{k}) \\
& =Z_{1}(\mathbf{k}) \tilde{\delta}_{\mathrm{m} L}(\mathbf{k})+\int \frac{\mathrm{d}^{3} \mathbf{k}_{1}}{(2 \pi)^{3}} \frac{\mathrm{d}^{3} \mathbf{k}_{2}}{(2 \pi)^{3}} Z_{2}\left(\mathbf{k}_{1}, \mathbf{k}_{2}\right) \tilde{\delta}_{\mathrm{m} L}\left(\mathbf{k}_{1}, t\right) \tilde{\delta}_{\mathrm{m} L}\left(\mathbf{k}_{2}, t\right)(2 \pi)^{3} \delta_{D}^{3}\left(\mathbf{k}_{12}-\mathbf{k}\right) .
\end{aligned}
$$


Using the standard Eulerian perturbation theory 40,42, where an irrotational, pressure-less single-fluid matter field is assumed, the kernels are given as

$$
\begin{aligned}
& Z_{1}(\mathbf{k}) \equiv b+f \mu^{2} \\
& Z_{2}\left(\mathbf{k}_{1}, \mathbf{k}_{2}\right) \equiv b F_{2}\left(\mathbf{k}_{1}, \mathbf{k}_{2}\right)+f \mu^{2} G_{2}\left(\mathbf{k}_{1}, \mathbf{k}_{2}\right)+\frac{f \mu k}{2}\left[\frac{\mu_{1}}{k_{1}}\left(b+f \mu_{2}^{2}\right)+\frac{\mu_{2}}{k_{2}}\left(b+f \mu_{1}^{2}\right)\right]
\end{aligned}
$$

where $\mathbf{k} \equiv \mathbf{k}_{1}+\mathbf{k}_{2}, \mu$ is the cosine angle between the wavevector $\mathbf{k}$ and the line-of-sight $\operatorname{direction}, \mu \equiv \hat{\mathbf{n}} \cdot \hat{\mathbf{k}}=k_{\|} / k$ ( $k_{\|}$is the component along the line-of-sight direction), $f \equiv \mathrm{d} \ln D / \mathrm{d} \ln a, D$ is the linear growth rate, and $b$ is the linear bias parameter of galaxies. The pioneer work for the RSD effect is given in Ref. [34, and see Refs. [43 46] for the extension to the higher-order terms. Throughout this paper we assume the linear galaxy bias to model how the real-space distribution of galaxies is related to that of matter. Although the effect of the large-scale tides could cause an additional biasing effect on the tracers [8, 47, 49], the effect on the power spectrum is of the order of $\mathcal{O}\left(\left(\delta_{\mathrm{m} L}\right)^{2}\right)$, compared with the $\mathcal{O}\left(\delta_{\mathrm{m} L}\right)$ effect in $b$, so we ignore the effect for simplicity. The kernels $F_{2}\left(\mathbf{k}_{1}, \mathbf{k}_{2}\right)$ and $G_{2}\left(\mathbf{k}_{1}, \mathbf{k}_{2}\right)$ are the second-order kernels for the density and velocity perturbations, given by Eqs. (45) and (46) in Ref. [7]:

$$
\begin{aligned}
& F_{2}\left(\mathbf{k}_{1}, \mathbf{k}_{2}\right)=\frac{5}{7}+\frac{1}{2}\left(\frac{1}{k_{1}^{2}}+\frac{1}{k_{2}^{2}}\right)\left(\mathbf{k}_{1} \cdot \mathbf{k}_{2}\right)+\frac{2}{7} \frac{\left(\mathbf{k}_{1} \cdot \mathbf{k}_{2}\right)^{2}}{k_{1}^{2} k_{2}^{2}} \\
& G_{2}\left(\mathbf{k}_{1}, \mathbf{k}_{2}\right)=\frac{3}{7}+\frac{1}{2}\left(\frac{1}{k_{1}^{2}}+\frac{1}{k_{2}^{2}}\right)\left(\mathbf{k}_{1} \cdot \mathbf{k}_{2}\right)+\frac{4}{7} \frac{\left(\mathbf{k}_{1} \cdot \mathbf{k}_{2}\right)^{2}}{k_{1}^{2} k_{2}^{2}} .
\end{aligned}
$$

\section{B. Derivation of the responses of redshift-space power spectrum to super-survey modes}

We now consider how super-survey modes affect the redshift-space power spectrum observed in a finite-volume survey. Following the discussion in Refs. [10, 30, in the presence of super-survey modes $\left(\delta_{\mathrm{b}}, \tau_{i j}\right)$ for a given survey realization, the "observed" redshift-space power spectrum is formally expressed as

$$
P_{s W}\left(\mathbf{k} ; \delta_{\mathrm{b}}, \tau_{i j}\right)=P_{s}(\mathbf{k})+\frac{\partial P_{s}(\mathbf{k})}{\partial \delta_{\mathrm{b}}} \delta_{\mathrm{b}}+\frac{\partial P_{s}(\mathbf{k})}{\partial \tau_{i j}} \tau_{i j}
$$

Here we omitted the dependence of $P_{s W}(\mathbf{k})$ on the line-of-sight direction, $\hat{\mathbf{n}}$, for notational simplicity and we explicitly denote that the observed spectrum $P_{s W}\left(\mathbf{k} ; \delta_{\mathrm{b}}, \tau_{i j}\right)$ depends on the super-survey modes of a given survey realization, and $P_{s}(\mathbf{k})$ is the power spectrum without the super-survey modes. The functions $\partial P_{s}(\mathbf{k}) / \partial \delta_{\mathrm{b}}$ and $\partial P_{s}(\mathbf{k}) / \partial \tau_{i j}$ are so-called "response" functions describing a response of the redshift-space power spectrum to the super-survey modes via mode couplings in the nonlinear structure formation.

Now we derive the response function using the perturbation theory. The simplest way to do this is considering the squeezed limit of the bispectrum that arises from correlations between two short modes and one long mode (corresponding to the super-survey modes) [50. More specifically, let us consider a correlation of $\hat{P}_{s W}(\mathbf{k})($ Eq. 4 ) with the large-scale matter density field, $\tilde{\delta}_{\mathrm{m} L}(\mathbf{q})$ (q is the long mode):

$$
\begin{aligned}
\left\langle\hat{P}_{s W}(\mathbf{k}) \tilde{\delta}_{\mathrm{m} L}(\mathbf{q})\right\rangle & =\frac{1}{V_{W}} \int_{\mathbf{k}^{\prime} \in \mathbf{k}} \frac{\mathrm{d}^{3} \mathbf{k}^{\prime}}{V_{\mathbf{k}}} \int \frac{\mathrm{d}^{3} \mathbf{q}_{1}}{(2 \pi)^{3}} \frac{\mathrm{d}^{3} \mathbf{q}_{2}}{(2 \pi)^{3}}\left\langle\tilde{\delta}_{s}\left(\mathbf{k}^{\prime}-\mathbf{q}_{1}\right) \tilde{\delta}_{s}\left(-\mathbf{k}^{\prime}-\mathbf{q}_{2}\right) \tilde{\delta}_{\mathrm{m} L}(\mathbf{q})\right\rangle \tilde{W}\left(\mathbf{q}_{1}\right) \tilde{W}\left(\mathbf{q}_{2}\right) \\
& =\frac{1}{V_{W}} \int_{\mathbf{k}^{\prime} \in \mathbf{k}} \frac{\mathrm{d}^{3} \mathbf{k}^{\prime}}{V_{\mathbf{k}}} \int \frac{\mathrm{d}^{3} \mathbf{q}_{1}}{(2 \pi)^{3}} \frac{\mathrm{d}^{3} \mathbf{q}_{2}}{(2 \pi)^{3}} B_{s s m}\left(\mathbf{k}^{\prime}-\mathbf{q}_{1},-\mathbf{k}^{\prime}-\mathbf{q}_{2}, \mathbf{q}\right)(2 \pi)^{3} \delta_{D}^{3}\left(\mathbf{q}_{12}-\mathbf{q}\right) \tilde{W}\left(\mathbf{q}_{1}\right) \tilde{W}\left(\mathbf{q}_{2}\right)
\end{aligned}
$$

where we have defined the bispectrum between the redshift-space density field and the real-space density field:

$$
\left\langle\tilde{\delta}_{s}\left(\mathbf{k}_{1}\right) \tilde{\delta}_{s}\left(\mathbf{k}_{2}\right) \tilde{\delta}_{\mathrm{m} L}(\mathbf{q})\right\rangle \equiv B_{s s \mathrm{~m}}\left(\mathbf{k}_{1}, \mathbf{k}_{2}, \mathbf{q}\right)(2 \pi)^{3} \delta_{D}^{3}\left(\mathbf{k}_{1}+\mathbf{k}_{2}+\mathbf{q}\right)
$$

For the case that $k \gg q_{1}, q_{2}, q$, the bispectrum in the above equation arises from so-called squeezed triangles where two sides are nearly equal and in opposite direction. To see this, we can make the variable changes $\mathbf{k}-\mathbf{q}_{1} \leftrightarrow \mathbf{k}$ and $\mathbf{q}_{1}+\mathbf{q}_{2} \leftrightarrow \mathbf{q}$ under the delta function condition $\mathbf{q}_{12}+\mathbf{q}=\mathbf{0}$ and the approximation that $k \ll q$. The bispectrum we are interested in reads

$$
\lim _{q \rightarrow 0} B_{s s \mathrm{~m}}(\mathbf{k},-\mathbf{k}-\mathbf{q}, \mathbf{q}) .
$$


In this limit, the triangle configuration describes how the redshift-space power spectrum $P_{s}(\mathbf{k})$ is modulated by the super-survey modes $\tilde{\delta}_{\mathrm{m} L}(\mathbf{q})$. For convenience of the following discussion, with the help of Eq. 19. we assume that the squeezed bispectrum can be described by the response of $P_{s}(\mathbf{k})$ to the super-survey modes as

$$
\lim _{q \rightarrow 0} B_{s s \mathrm{~m}}(\mathbf{k},-\mathbf{k}-\mathbf{q}, \mathbf{q}) \equiv\left[\frac{\partial P_{s}(\mathbf{k})}{\partial \delta_{b}}+\left(\hat{q}_{i} \hat{q}_{j}-\frac{\delta_{i j}^{K}}{3}\right) \frac{\partial P_{s}(\mathbf{k})}{\partial \tau_{i j}}\right] P^{L}(q) .
$$

From Eq. (23), we can derive the response function $\partial P_{s}(\mathbf{k}) / \partial \delta_{\mathrm{b}}$ from the angle average of the squeezed bispectrum over $\mathrm{d}^{3} \mathbf{q}$ as

$$
\frac{\partial P_{s}(\mathbf{k})}{\partial \delta_{b}} P^{L}(q) \simeq \lim _{q \rightarrow 0} \int \frac{\mathrm{d} \Omega_{\mathbf{q}}}{4 \pi} B_{s s m}(\mathbf{k},-\mathbf{k}-\mathbf{q}, \mathbf{q})
$$

With this derivation, the response to the large-scale tide, $\partial P_{s}(\mathbf{k}) / \partial \tau_{i j}$ can be found from

$$
\frac{\partial P_{s}(\mathbf{k})}{\partial \tau_{i j}} \longleftarrow \text { coefficients in }\left(\hat{q}_{i} \hat{q}_{j}-\frac{\delta_{i j}^{K}}{3}\right) P^{L}(q) \text { in } \lim _{q \rightarrow 0} B_{s s \mathrm{~m}}(\mathbf{k},-\mathbf{k}-\mathbf{q}, \mathbf{q}) .
$$

Using the perturbation theory ansatz for $\tilde{\delta}_{s}(\mathbf{k})$ (Eq. 16) and assuming that the large-scale mode $\tilde{\delta}_{\mathrm{m} L}(\mathbf{q})$ is in the linear regime, the leading-order contribution of the squeezed bispectrum can be expressed in terms of the modecoupling kernels as

$$
B_{s s m}(\mathbf{k},-\mathbf{k}-\mathbf{q}, \mathbf{q}) \simeq 2 Z_{1}(\mathbf{k}+\mathbf{q}) Z_{2}(\mathbf{k}+\mathbf{q},-\mathbf{q}) P^{L}(|\mathbf{k}+\mathbf{q}|) P^{L}(q)+2 Z_{1}(\mathbf{k}) Z_{2}(\mathbf{k}, \mathbf{q}) P^{L}(k) P^{L}(q) .
$$

Inserting Eq. (17) into Eq. 26) and using the relations (Eq. 24 and Eq. 25), we can find that the response functions for the redshift-space power spectrum are

$$
\begin{aligned}
\frac{\partial P_{s}(\mathbf{k})}{\partial \delta_{\mathrm{b}}}= & {\left[\frac{47}{21}-\frac{1}{3} \frac{\mathrm{d} \ln P^{L}(k)}{\mathrm{d} \ln k}\right] b^{2} P^{L}(k)+\left[\frac{b}{3}+\mu^{2}\left(\frac{26}{7}+2 b\right)-\frac{\mu^{2}}{3}(2+b) \frac{\mathrm{d} \ln P^{L}(k)}{\mathrm{d} \ln k}\right] b f P^{L}(k) } \\
& +\left[\frac{1}{21}(31+70 b)-\frac{1}{3}(1+2 b) \frac{\mathrm{d} \ln P^{L}(k)}{\mathrm{d} \ln k}\right] f^{2} \mu^{4} P^{L}(k)+\left[\frac{1}{3}\left(4 \mu^{2}-1\right)-\frac{\mu^{2}}{3} \frac{\mathrm{d} \ln P^{L}(k)}{\mathrm{d} \ln k}\right] f^{3} \mu^{4} P^{L}(k),
\end{aligned}
$$

and

$$
\begin{aligned}
\frac{\partial P_{s}(\mathbf{k})}{\partial \tau_{i j}}= & {\left[\frac{8}{7} \hat{k}_{i} \hat{k}_{j}-\hat{k}_{i} \hat{k}_{j} \frac{\mathrm{d} \ln P^{L}(k)}{\mathrm{d} \ln k}\right] b^{2} P^{L}(k) } \\
& +\left[b \hat{n}_{i} \hat{n}_{j}+\frac{24}{7} \mu^{2} \hat{k}_{i} \hat{k}_{j}-\mu\left(2 \mu \hat{k}_{i} \hat{k}_{j}+b h_{i j}\right) \frac{\mathrm{d} \ln P^{L}(k)}{\mathrm{d} \ln k}\right] b f P^{L}(k) \\
& +\left[\frac{16}{7} \mu \hat{k}_{i} \hat{k}_{j}+4 b h_{i j}-\left(\mu \hat{k}_{i} \hat{k}_{j}+2 b h_{i j}\right) \frac{\mathrm{d} \ln P^{L}(k)}{\mathrm{d} \ln k}\right] f^{2} \mu^{3} P^{L}(k) \\
& +\left[\left(4 \mu h_{i j}-\hat{n}_{i} \hat{n}_{j}\right)-\mu h_{i j} \frac{\mathrm{d} \ln P^{L}(k)}{\mathrm{d} \ln k}\right] f^{3} \mu^{4} P^{L}(k),
\end{aligned}
$$

where

$$
h_{i j} \equiv \hat{k}_{(i} \hat{n}_{j)}=\frac{1}{2}\left(\hat{k}_{i} \hat{n}_{j}+\hat{k}_{j} \hat{n}_{i}\right) .
$$

These are full expressions of the responses of redshift-space power spectrum to the large-scale perturbations. Compared with the results in Ref. [30, there are additional effects of the super-survey modes on the redshift-space power spectrum, that is, there are terms including the couplings between the large-scale tides $\tau_{i j}$ and the line-of-sight direction $\hat{\mathbf{n}}$ as expected. The response function for $\delta_{\mathrm{b}}, \partial P_{s}(\mathbf{k}) / \partial \delta_{\mathrm{b}}$, agrees with Eq. (65) in Ref. [51] if we set $b=1$ in the above equation. The response functions, $\partial P_{s}(\mathbf{k}) / \partial \delta_{\mathrm{b}}$ and $\partial P_{s}(\mathbf{k}) / \partial \tau_{i j}$, show several effects caused by the super-survey modes. First, the large-scale perturbations could speed up or slow down the growth of short modes: for example, if the large-scale tide along a particular direction is positive, say $\tau_{i i}>0$, the expansion of a local volume along the direction is slower than that of the global universe, so the growth of short modes with $\mathbf{k}$ along the direction can be enhanced. Secondly, the super-survey modes cause a dilation of the comoving wavelengths. Because the large-scale perturbations can be realized as a modification of the local expansion, the comoving wavelengths which 
an observer infer are modulated by the super-survey modes, which imprints a modulation in the power spectrum. Thirdly, the super-survey modes alter the peculiar velocities through the effects on the gravitational force, so alter the redshift-space distortion effects along the line-of-sight direction. Here the large-scale tides cause modifications in the clustering pattern along all the three directions.

In the following we focus on the response function for $\tau_{i j}$, and we do not consider the response for $\delta_{\mathrm{b}}$. From Eq. 28 we can find several types of anisotropies in the redshift-space power spectrum: the standard RSD effect $\mu^{2}=\hat{k}_{i} \hat{k}_{j} \hat{n}_{i} \hat{n}_{j}$ (Kaiser factor), and the effects due to $\tau_{i j}$ that have dependences of $\tau_{i j} \hat{k}_{i} \hat{k}_{j}, \tau_{i j} \hat{k}_{i} \hat{n}_{j}$, and $\tau_{i j} \hat{n}_{i} \hat{n}_{j}$, respectively. First, let us remind of the physical origin of the Kaiser factor. It comes from $\partial_{i} v_{j} \hat{n}_{i} \hat{n}_{j}(\operatorname{see}$ Eq. 14 ). This means that the Kaiser anisotropy reflects the projection of the velocity $\operatorname{shear}\left(\partial_{(i} v_{j}\right)$, in Fourier space $\left.\propto \hat{k}_{i} \hat{k}_{j}\right)$ onto the line-of-sight direction. In other words, since the velocity shear corresponds to the tidal field, the Kaiser factor can be interpreted as the projection of the short-mode tidal field onto the line-of-sight direction. Next, the terms proportional to $\tau_{i j} \hat{k}_{i} \hat{k}_{j}$ represent a coupling between the large-scale tides $\tau_{i j}$ and the small-scale tides, where the latter has directional dependences given by $\propto\left(\hat{k}_{i} \hat{k}_{j}-\frac{1}{3} \delta_{i j}^{K}\right)$. The terms of $\tau_{i j} \hat{n}_{i} \hat{n}_{j}$ are like the Kaiser factor, that is, the projection of the large-scale tides $\tau_{i j}$ onto the line-of-sight direction. Note that the terms proportional to $h_{i j}$ always appear with $\mu=\hat{\mathbf{k}} \cdot \hat{\mathbf{n}}$, because of the parity invariance of the power spectrum, i.e. $P_{s}(\mathbf{k})=P_{s}(-\mathbf{k})$. Then, $\tau_{i j} \hat{k}_{i} \hat{n}_{j} \mu=\tau_{i j} \hat{k}_{i} \hat{k}_{l} \hat{n}_{j} \hat{n}_{l}$ is a consequence of the projection of the coupling between the large-scale tides $\tau_{i j}$ and the small-scale velocity $\propto \hat{k}_{i}$ onto the line-of-sight direction.

\section{The large-scale mode effects on the two-dimensional redshift-space power spectrum: $P_{s}^{2 \mathrm{D}}\left(k_{\|}, k_{\perp}\right)$}

The main purpose of this paper is to estimate the impact of super-survey modes on the RSD measurements as well as Alcock-Paczynski (AP) test [36] through a measurements of the redshift-space power spectrum. To do this, we employ the standard approach used in an analysis of the redshift-space power spectrum. Since the RSD effect is only along the line-of-sight direction and does not affect the clustering pattern in the two-dimensional plane perpendicular to the line-of-sight direction, a usual way to measure the redshift-space power spectrum is making the angle average given as

$$
P_{s W}^{2 \mathrm{D}}\left(k_{\|}, k_{\perp} ; \tau_{i j}\right) \equiv \int_{0}^{2 \pi} \frac{\mathrm{d} \varphi_{\mathbf{k}_{\perp}}}{2 \pi} P_{s W}\left(\mathbf{k} ; \tau_{i j}\right),
$$

where we have set the line-of-sight direction as $z$-axis, $\hat{n}_{i}=\delta_{i z}^{K}$ and used the decomposition of wavevector, $\mathbf{k}=$ $\left(k_{\perp} \cos \varphi_{\mathbf{k}_{\perp}}, k_{\perp} \sin \varphi_{\mathbf{k}_{\perp}}, k_{\|}\right)$with the conditions $\left(k_{\perp}, k_{\|}\right)=k\left(\sqrt{1-\mu^{2}}, \mu\right)$.

By inserting Eqs.19) and (28) into Eq. (30) we can find

$$
\begin{aligned}
P_{s W}^{2 \mathrm{D}}\left(k_{\perp}, k_{\|} ; \tau_{i j}\right)= & \left(b+f \mu^{2}\right)^{2} P^{L}(k)+\left[\frac{8}{7} b^{2} P^{L}(k)-b^{2} \frac{\mathrm{d} P^{L}(k)}{\mathrm{d} \ln k}\right] \frac{3 \mu^{2}-1}{2} \tau_{33} \\
& +f b\left[\left\{b+\frac{12}{7} \mu^{2}\left(3 \mu^{2}-1\right)\right\} P^{L}(k)-\mu^{2}\left\{b+\left(3 \mu^{2}-1\right)\right\} \frac{\mathrm{d} P^{L}(k)}{\mathrm{d} \ln k}\right] \tau_{33} \\
& +f^{2} \mu^{4}\left[\left\{4 b+\frac{8}{7}\left(3 \mu^{2}-1\right)\right\} P^{L}(k)-\left(2 b+\frac{3 \mu^{2}-1}{2}\right) \frac{\mathrm{d} P^{L}(k)}{\mathrm{d} \ln k}\right] \tau_{33} \\
& +f^{3} \mu^{4}\left[\left(4 \mu^{2}-1\right) P^{L}(k)-\mu^{2} \frac{\mathrm{d} P^{L}(k)}{\mathrm{d} \ln k}\right] \tau_{33},
\end{aligned}
$$

where we have used the following identities under the presence of the line-of-sight direction

$$
\begin{aligned}
& \int_{0}^{2 \pi} \frac{\mathrm{d} \phi_{\mathbf{k}_{\perp}}}{2 \pi} \hat{k}_{i} \hat{k}_{j}=\frac{1-\mu^{2}}{2} \delta_{i j}^{K}+\frac{3 \mu^{2}-1}{2} \hat{n}_{i} \hat{n}_{j}, \\
& \int_{0}^{2 \pi} \frac{\mathrm{d} \phi_{\mathbf{k}_{\perp}}}{2 \pi} \hat{k}_{i}=\mu \hat{n}_{i},
\end{aligned}
$$

with the trace-less condition of $\tau_{i j}$, i.e. $\tau_{i j} \delta_{i j}^{K}=0$. Eq. 31 is one of the main results of this paper. The equation shows that the large-scale tides cause an additional anisotropic clustering in the two-dimensional redshift-space power spectrum in addition to the Kaiser distortion. The amount of the distortion depends on the line-of-sight component of the tides, $\tau_{33}$, in a given survey realization. The tides cause anisotropic distortions up to the order of $\mu^{6}$, while 
the standard Kaiser RSD effect causes distortions up to $\mu^{4}$. Thus the large-scale tides in a given survey realization cause a bias in the redshift-space power spectrum. There are two ways to take into account the effect. One way is to include the effect as an additional noise in the error covariance matrix of the power spectrum as studied in Ref. 30. Alternative approach, which we take in this paper, is to treat the effect as a signal rather than noise. We can model this effect by treating the bias as a purely systematic additive shift in the redshift-space power spectrum, where an amount of the bias is given by the power spectrum response multiplied by a free parameter $\tau_{33}$. Then we can use the measured power spectrum to infer the $\tau_{33}$ value in the survey realization. We will study how a large-volume galaxy redshift survey can constrain the large-scale tides and also how it could cause a degradation in cosmological parameters.

\section{THE IMPACT OF LARGE-SCALE TIDAL EFFECT ON REDSHIFT-SPACE POWER SPECTRUM}

\section{A. Fisher information matrix}

In this section, following Refs. 37] and 38, we study how the large-scale tides affect the BAO and RSD measurements in the redshift-space power spectrum [2], based on the Fisher information matrix formalism.

The two-point correlation function of galaxies is measured as a function of the separation lengths between paired galaxies. To measure this separation, the position of each galaxy needs to be inferred from the measured redshift and angular position. Then the separation lengths perpendicular and parallel to the line-of-sight direction from the measured quantities are given as $r_{\perp} \propto \Delta \theta$ and $r_{\|} \propto \Delta z$, with $\Delta \theta$ and $\Delta z$ being the differences between the angular positions and the redshifts of the paired galaxies. To convert the observables $(\Delta \theta, \Delta z)$ to the quantities $\left(r_{\perp}, r_{\|}\right)$, one has to assume a reference cosmological model. Considering this transformation, the wavenumbers are given as

$$
k_{\perp, \mathrm{ref}}=\frac{D_{A}(z)}{D_{A, \mathrm{ref}}(z)} k_{\perp}, \quad k_{\|, \mathrm{ref}}=\frac{H_{\mathrm{ref}}(z)}{H(z)} k_{\|},
$$

where $D_{A}(z)$ is the angular diameter distance and $H(z)$ is the Hubble expansion rate. The quantities with subscript "ref" mean the quantities for an assumed "reference" cosmological model, and the quantities without the subscript mean the underlying true values. Since the reference cosmological model we assume generally differs from the underlying true cosmology, an apparent geometrical distortion is caused in the two-dimensional pattern of galaxy clustering. In principle, this distortion could be measured using only the isotropy of clustering statistics, the so-called AlcockPaczynski (AP) test [36], but a more robust measurement of both $D_{A}(z)$ and $H(z)$ can be obtained by searching for the "common" BAO scales in the pattern of galaxy clustering, as the standard ruler, in combination with the CMB constraints [37, 38].

We will use the currently standard $\Lambda \mathrm{CDM}$ model as a guidance for the parameter dependence of our constraints and as an effective realistic description of the galaxy clustering. To be more quantitative, we assume that the redshift-space galaxy power spectrum measured from a hypothetical survey realization is given in the linear regime as

$$
P_{s W, \mathrm{ref}}^{2 \mathrm{D}, \mathrm{obs}}\left(k_{\|, \mathrm{ref}}, k_{\perp, \mathrm{ref}} ; \tau_{33}\right)=\frac{D_{A, \mathrm{ref}}^{2} H}{D_{A}^{2} H_{\mathrm{ref}}} P_{s W}^{2 \mathrm{D}}\left(k_{\|}, k_{\perp} ; \tau_{33}\right)+P_{\mathrm{sn}},
$$

where $P_{s W}^{2 \mathrm{D}, \text { obs }}$ is the "observed" or "estimated" power spectrum from a given survey realization, $P_{s W}^{2 \mathrm{D}}$ on the righthand side is the true, underlying true power spectrum (Eq. 31), measured if an observer employs the true cosmological model, and $P_{\mathrm{sn}}$ is a parameter (constant number) to model a possible contamination of a residual shot noise to the power spectrum measurement.

To make the parameter forecast, we employ the method developed in Ref. 2, 3, 52. Assuming that the redshift-space power spectrum is measured from a hypothetical survey volume the Fisher information matrix of model parameters can be computed as

$$
\begin{array}{rl}
F_{\alpha \beta}^{\text {galaxy }}=\int_{-1}^{1} d & d \mu \int_{k_{\min }}^{k_{\max }} \frac{2 \pi k^{2} \mathrm{~d} k}{2(2 \pi)^{3}} \frac{\partial \ln P_{s W, \text { ref }}^{2 \mathrm{D}, \text { obs }}\left(k, \mu ; z_{i}\right)}{\partial p_{\alpha}} \frac{\partial \ln P_{s W, \text { ref }}^{2 \mathrm{D}, \mathrm{obs}}\left(k, \mu ; z_{i}\right)}{\partial p_{\beta}} \\
& \times V_{\mathrm{eff}}\left(k ; z_{i}\right) \exp \left[-k^{2} \Sigma_{\perp}^{2}-k^{2} \mu^{2}\left(\Sigma_{\|}^{2}-\Sigma_{\perp}^{2}\right)\right]
\end{array}
$$

where $\partial P_{s W \text {,ref }}^{2 \mathrm{D}, \mathrm{obs}} / \partial p_{\alpha}$ is the partial derivative of the galaxy power spectrum (Eq. 34 with respect to the $\alpha$-th parameter around the reference cosmological model. The effective survey volume $V_{\text {eff }}$ and the Lagrangian displacement fields $\Sigma_{\|}$ 
and $\Sigma$ to model the smearing effect are given as

$$
\begin{aligned}
V_{\text {eff }}\left(k, \mu ; z_{i}\right) & \equiv\left[\frac{\bar{n}_{g}\left(z_{i}\right) P_{s W}^{2 \mathrm{D}}\left(k, \mu ; z_{i}\right)}{\bar{n}_{g}\left(z_{i}\right) P_{s W}^{2 \mathrm{D}}\left(k, \mu ; z_{i}\right)+1}\right]^{2} V_{\text {survey }}\left(z_{i}\right), \\
\Sigma_{\perp}(z) & \equiv c_{\mathrm{rec}} D(z) \Sigma_{0} \\
\Sigma_{\|}(z) & \equiv c_{\mathrm{rec}} D(z)\left(1+f_{g}\right) \Sigma_{0} .
\end{aligned}
$$

Here $V_{\text {survey }}\left(z_{i}\right)$ is the comoving volume of the redshift slice centered at $z_{i}, \Sigma_{0}$ is the present-day Lagrangian displacement field, given as $\Sigma_{0}=11 h^{-1} \mathrm{Mpc}$ for $\sigma_{8}=0.8$ [53], and the parameter $c_{\text {rec }}$ is a parameter to model the reconstruction method of the BAO peaks (see below). In Eq. 35], we take the exponential factor of the smearing effect outside of the derivatives of $P_{s W \text {,ref }}^{2 \mathrm{D} \text {, obs }}$. This is equivalent to marginalizing over uncertainties in $\Sigma_{\|}$and $\Sigma_{\perp}$. We include the parameter for the large-scale tides for the survey volume, i.e. $\tau_{33}$ in addition to the cosmological parameters, the distances in each redshift slice, and other nuisance parameters:

$$
p_{\alpha}=\left\{\tau_{33}, \Omega_{\mathrm{m} 0}, A_{s}, n_{s}, \alpha_{s}, \Omega_{\mathrm{m} 0} h^{2}, \Omega_{\mathrm{b} 0} h^{2}, D_{A}\left(z_{i}\right), H\left(z_{i}\right), b_{g}\left(z_{i}\right), \beta\left(z_{i}\right), P_{\mathrm{sn}}\left(z_{i}\right)\right\},
$$

where $A_{s}, n_{s}$ and $\alpha_{s}$ are parameters of the primordial power spectrum; $A_{s}$ is the amplitude of the primordial curvature perturbation, and $n_{s}$ and $\alpha_{s}$ are the spectral tilt and the running spectral index. The set of cosmological parameters determines the shape of the linear power spectrum. For the $k$-integration, we set $k_{\min }=10^{-4} h / \mathrm{Mpc}$ and $k_{\max }=0.5 \mathrm{~h} / \mathrm{Mpc}$, but the exponential factor in Eq. (35) suppresses the information from the nonlinear scales. The Fisher parameter forecasts depend on the reference cosmological model for which we assumed the model consistent with the WMAP 7-year data [54]. In this paper, we consider a single redshift slice, and then consider 12 parameters in total in the Fisher analysis.

Furthermore, we assume the BAO reconstruction method in Ref. 53. Because the large-scale peculiar velocity field of galaxies in large-scale structure can be inferred from the measured galaxy distribution, the inferred velocity field allows for pulling back each galaxy to its position at an earlier epoch and then reconstructing the galaxy distribution more in the linear regime. As a result, one can correct to some extent the smearing effect in Eq. (35) and sharpen the BAO peaks in the galaxy power spectrum. Padmanabhan et al. [55] implemented this method to the real data, SDSS DR7 LRG catalog, and showed that the reconstruction method can improve the distance error by a factor of 2 . The improvement was equivalent to reducing the nonlinear smoothing scale from 8.1 to $\Sigma_{\mathrm{nl}}=4.4 h^{-1} \mathrm{Mpc}$, about a factor of 2 reduce in the displacement field. In the Fisher matrix calculation, we used $c_{\text {rec }}=0.5$ as a default choice [55].

In the following forecast, we assume the $\mathrm{BAO}$ experiment combined with the CMB constraints expected from the Planck satellite:

$$
\boldsymbol{F}=\boldsymbol{F}^{\mathrm{CMB}}+\boldsymbol{F}^{\text {galaxy }}
$$

where $\boldsymbol{F}_{\mathrm{CMB}}$ is the Fisher matrix for the CMB measurements. We employ the method in Ref. [2] to compute the CMB Fisher matrix, where we assumed the standard $\Lambda \mathrm{CDM}$ model for the physics prior to recombination that determines the sound horizon scale or the BAO scale.

\section{B. Results}

As a working example, we consider a hypothetical survey that is characterized by the central redshift $z=0.5$, the comoving volume $V=1(\mathrm{Gpc} / h)^{3}$, the mean number density of galaxies $\bar{n}_{g}=10^{-3}(h / \mathrm{Mpc})^{3}$ and linear bias parameter $b=2$, respectively. For simplicity we consider a single redshift slice. In reality, when a galaxy redshift survey probes galaxies over a wide range of redshifts, one can use the clustering analysis in multiple redshift slices and then combine their cosmological information.

In Fig. 1 we show the marginalized 68\% CL error contours in each of two-dimensional sub-space that include either two of the large-scale tidal parameter, $\tau_{33}$, the distance parameters, $D_{A}$ or $H$, or the RSD parameter $\beta$, where the contours include marginalization over other parameters. Note that $\tau_{33}$ has little degeneracy with other parameters. More quantitatively, the cross-correlation coefficients defined as $c_{i j}=\left(F^{-1}\right)_{i j} / \sqrt{\left(F^{-1}\right)_{i i}\left(F^{-1}\right)_{j j}}$ with $i=\tau_{33}$, after the CMB Fisher matrix is added, is almost unity for either one of these three parameters is taken for $j$, while the cross-coefficients are smaller for other parameters, less than $\mathcal{O}(0.2)$. The contours in each panel of Fig. 1 show how an uncertainty in $\tau_{33}$ causes a degeneracy with estimation of other parameter. Since the large-scale tides cause apparent anisotropies in the observed clustering of galaxies as the radial AP anisotropy and the RSD effect do, allowing $\tau_{33}$ to freely vary in the parameter estimation causes significant degeneracies with $\beta$ and $H$. The degeneracy between $\tau_{33}$ and $D_{A}$ arises from the trace-less nature of $\tau_{i j}$; changing $\tau_{33}$ leads to a change in $\tau_{11}+\tau_{22}\left(=-\tau_{33}\right)$ and therefore causes an apparent distortion in the $k_{\perp}$-direction, which mimics the cosmological distortion due to a change in $D_{A}$. 


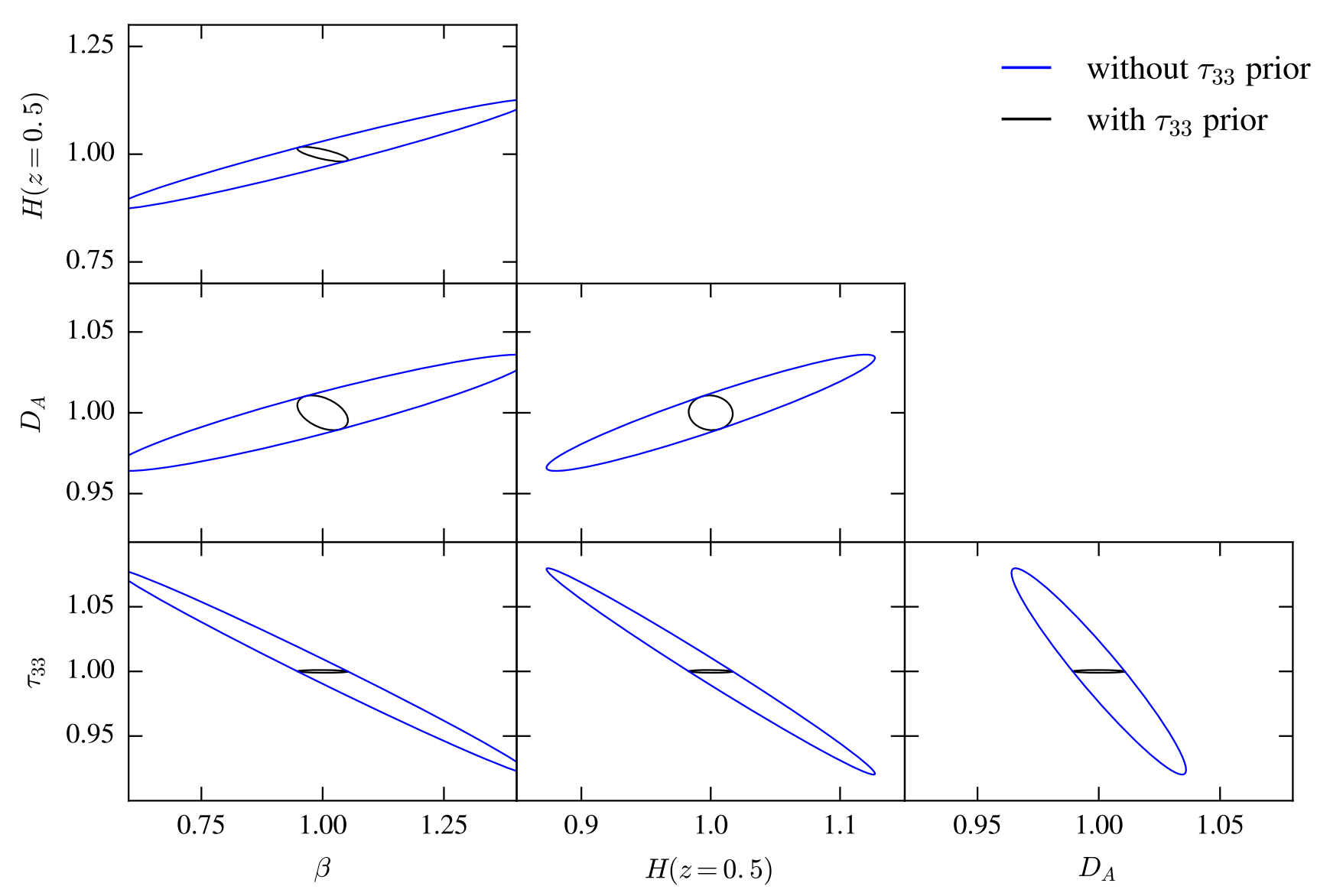

FIG. 1. $68 \%$ CL error ellipse for the parameters, $\tau_{33}, D_{A}, H$ and $\beta$, including marginalization over other parameters in the Fisher analysis (see Section IV A for details). The inner black contour in each panel shows the result when $\sigma_{\tau_{33}}=1.04 \times 10^{-3}$ is employed as the $\tau_{33}$ prior, which is taken from the rms value expected for the $\Lambda$ CDM model and the assumed galaxy survey that is characterized by $V=1(\mathrm{Gpc} / h)^{3}, \bar{n}_{\mathrm{g}}=10^{-3}(h / \mathrm{Mpc})^{3}$ and $b=2$.

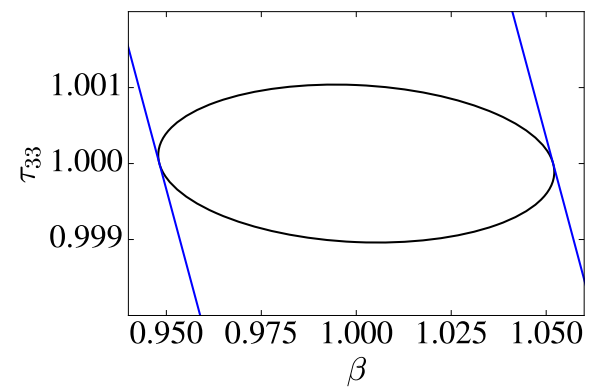

Fisher ellipse for $\tau_{33}$ and $\beta$

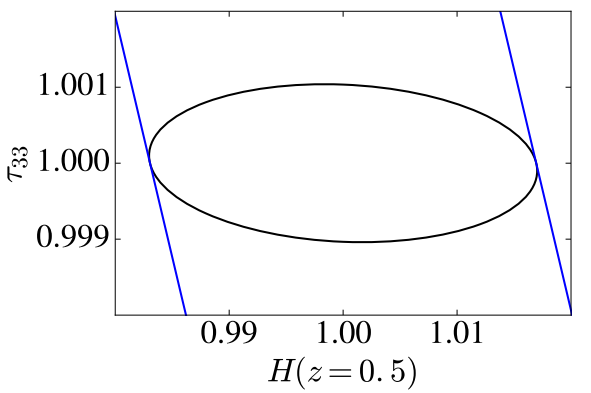

Fisher ellipse for $\tau_{33}$ and $H$

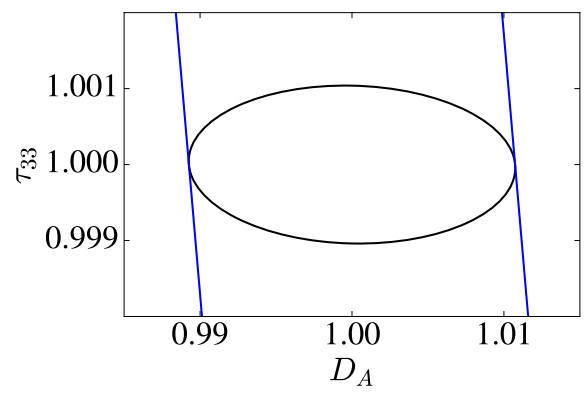

Fisher ellipse for $\tau_{33}$ and $D_{A}$

FIG. 2. A zoom-in version of Fig. 1, around the fiducial model for the Fisher analysis.

However, if adding the prior on $\tau_{33}$ assuming the $\Lambda \mathrm{CDM}$ model, i.e. $\tau_{33}=0$ for the expectation value and $\sigma_{\tau_{33}}=1.04 \times 10^{-3}$ for the $\mathrm{rms}$ value for $V=1(\mathrm{Gpc} / h)^{3}$, it lifts the degeneracies, recovering a high-precision measurement for each cosmological parameter. Fig. 2 shows a zoom-in version of the contours around the central value (the input model in the Fisher analysis), and shows that the prior of $\tau_{33}$ efficiently breaks the parameter degeneracies. In particular, even if an actual value of $\tau_{33}$ in a given survey realization is off from zero by more than a few $\sigma_{\tau_{33}}$, it does not seem to cause a significant bias in the parameters.

Nevertheless it is interesting to ask whether a measurement of redshift-shift power spectrum of galaxies can be 


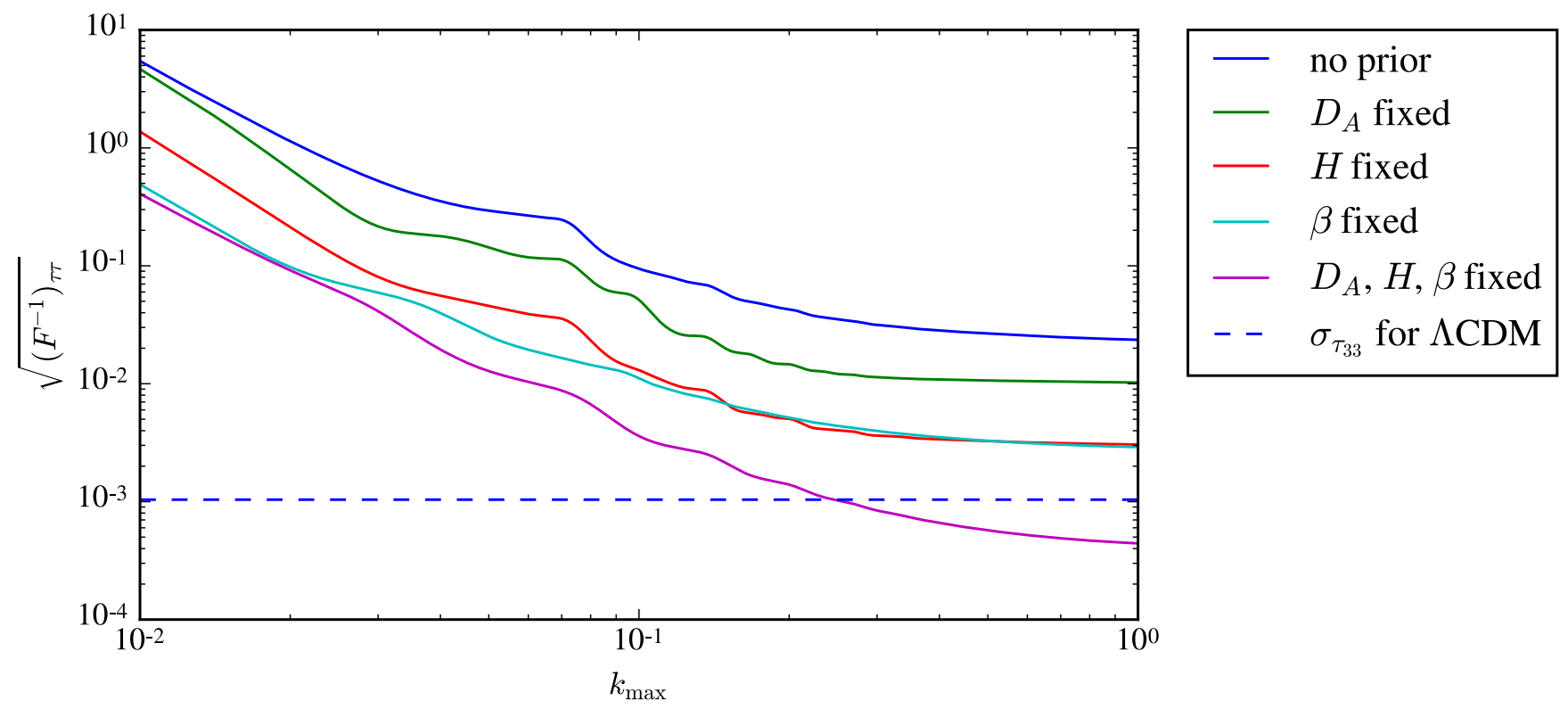

FIG. 3. The marginalized error on the estimation of $\tau_{33}, \sqrt{\left(F^{-1}\right)_{\tau \tau}}$, as a function of the maximum wavenumber $k_{\max }$ up to which the redshift-space power spectrum information is included in the Fisher analysis (see text for the details). The different solid curves show the results when any prior on other parameters $\left(D_{A}, H\right.$ and $\left.\beta\right)$ are not employed or when some or all the parameters are fixed to their values for the $\Lambda \mathrm{CDM}$ model. The horizontal dashed curve is the rms value, $\sigma_{\tau_{33}}$, expected for the $\Lambda \mathrm{CDM}$ model and the survey volume. Note that we did not impose any prior on other parameters (Eq. 39), although the CMB information is added.

used to constrain the large-scale tides, $\tau_{33}$, rather than employing the prior, if one can include the information up to the larger $k$ beyond the weakly nonlinear regime. To address this possibility, we need to know the response of the redshift-space power spectrum to the tides, $\partial P_{s}(\mathbf{k}) / \partial \tau_{33}$, in the nonlinear regime where the perturbation theory breaks down. To estimate the response function in the nonlinear regime requires to, e.g. use a separate universe simulation where the large-scale tidal effect is included in the background expansion, similarly to the method used for estimating the response for the mean density modulation, $\partial P_{s} / \partial \delta_{\mathrm{b}}$, in Refs. [21, 22, 56, 57. This is beyond the scope of this paper, so here we simply assume that the response function derived using the perturbation theory holds in the nonlinear regime. This would be conservative, because the response is likely to be amplified in the nonlinear regime as shown in Ref. 21. Furthermore, to include the effect of the large-scale tides up to the nonlinear regime, we set $\Sigma=0$ for the BAO smearing factor in the Fisher analysis. In practice, the smearing factor also depends on nonlinear structure formation, and therefore would depend on $\tau_{33}$. This is a simplified assumption, but we believe that the following result gives a rough estimation of the genuine effect. Fig. 3 shows how an accuracy of the $\tau_{33}$ estimation is improved when including the redshift-space power spectrum information up to a given maximum wavenumber $k_{\text {max }}$. Without any prior, $\tau_{33}$ is estimated to about $1 \%$ accuracy for a survey volume of $V=1(\mathrm{Gpc} / h)^{3}$. When fixing other parameters to their values for $\Lambda \mathrm{CDM}$ model, the accuracy of $\tau_{33}$ is dramatically improved. In particular, when all the distortion parameters, $D_{A}, H$ and $\beta$, are fixed, the $\tau_{33}$ parameter could be determined to an accuracy better than the rms for $\Lambda$ CDM model, if the redshift-space power spectrum information is included up to $k_{\max } \gtrsim 0.25 h / \mathrm{Mpc}$. This result implies that the anisotropic clustering information in such a nonlinear regime could be used to infer the large-scale tides for a given survey realization.

\section{DISCUSSION}

In this paper, using the standard perturbation theory, we derived the response functions of the redshift-space power spectrum to the super-survey modes, both the isotropic component, $\partial P_{s}(\mathbf{k}) / \partial \delta_{\mathrm{b}}$, and the anisotropic components, $\partial P_{s}(\mathbf{k}) / \partial \tau_{i j}$. Since a given survey realization is generally embedded in the presence of super-survey modes, $\delta_{\mathrm{b}}$ and $\tau_{i j}$, that are not direct observables in a finite-volume survey, it is important to take into account the response functions which describe how the super-survey modes cause a modulation in the redshift-space power spectrum measured in 
the survey volume, compared with the ensemble average expectation for an infinite volume. There are two effects. First, the presence of the super-survey modes changes the growth of small-scale fluctuations via the nonlinear mode coupling. Secondly, it causes a dilation effect, the modulation of a short distance scale due to the change of the local expansion factor in the finite volume region. In particular we showed that the large-scale tides, $\tau_{i j}$, cause an apparent anisotropic clustering in the redshift-space power spectrum, where the effect has directional dependence determined by an alignment of the large-scale tides, the directions of small-scale modes, and the line-of-sight direction. This effect mimics an anisotropic clustering due to the redshift-space distortion effect of the small-scale peculiar velocities of galaxies as well as the apparent cosmological distortion caused by the use of an incorrect cosmological model in the clustering analysis.

To assess a possible impact of $\tau_{i j}$ on parameter estimation from a measurement of the redshift-space power spectrum in a given survey realization, we used the Fisher information matrix formalism. To do this, we treated the effect of $\tau_{i j}$ as a "signal" rather than an additional source of statistical errors in the redshift-space power spectrum measurement, because it causes a modulation in the measured power spectrum as do cosmological parameters around the true model: $P_{s}\left(\mathbf{k} ; \tau_{i j}\right)=P_{s}(\mathbf{k})+\tau_{i j} \partial P_{s}(\mathbf{k}) / \partial \tau_{i j}$, where the tensor $\tau_{i j}$ takes particular values in a given survey realization. Thus as long as an accurate model of the response function is given as a function of cosmological models, it would be straightforward to include the effect in parameter estimation. In this paper, we considered the two-dimensional redshift-space power spectrum, $P_{s}^{2 \mathrm{D}}\left(k_{\perp}, k_{\|} ; \tau_{33}\right)$ as an observable, which is obtained from the azimuthal angle average of the redshift-space power spectrum estimator in the two-dimensional plane perpendicular to the line-of-sight direction under the distant observer approximation. In this case, the effects of the large-scale tides are modeled by a single quantity, $\tau_{33}$, the line-of-sight component of the tides. We showed that, if allowing $\tau_{33}$ to freely vary, it causes a significant degradation in the parameters, $D_{A}, H$ and $\beta$, due to almost perfect degeneracies between $\tau_{33}$ and the parameters in the power spectrum. If one adopts a prior on $\tau_{33}$ assuming the rms expected for a $\Lambda$ CDM model, it efficiently lifts the parameter degeneracies and restores an accuracy of the cosmological parameters that are expected for a galaxy survey without the super-survey mode. Thus the impact of the large-scale tides on the redshift-space power spectrum is not as large as the impact of the large-scale density contrast, $\delta_{\mathrm{b}}$, on a real-space power spectrum such as the weak lensing power spectrum [10, 21, as long as the large-scale tides obey the $\Lambda$ CDM expectation. The reason for this less-significant impact is partly because the statistical uncertainty in a measurement of the quadrupole power spectrum, which is the lowest-order observable to extract the redshift-space distortion, is dominated by the statistical uncertainty in the monopole power spectrum measurement [30].

We have also addressed whether a measurement of redshift-space power spectrum can be used to constrain $\tau_{33}$ in the survey realization, rather than treating $\tau_{33}$ as a nuisance parameter. Because the presence of $\tau_{33}$ causes a mode-coupling with all the small-scale fluctuations, we showed that $\tau_{33}$ can be well constrained at an accuracy better than the rms for a $\Lambda \mathrm{CDM}$ model, if we can use the redshift-space power spectrum information up to small scales, $k_{\max } \gtrsim 0.25 h / \mathrm{Mpc}$ and if the cosmological parameters including $D_{A}, H$ and $\beta$ are sufficiently well constrained, e.g. by other cosmological probes. This is an interesting possibility, because the method gives an access to such large-scale tides from a measurement of the small-scale fluctuations, and the large-scale tides would contain the information on physics in the early universe, e.g. statistical anisotropies arising from the inflation physics [58] or a large-scale anisotropy due to the super-curvature fluctuation [59].

However, there are several limitations in the results shown in this paper. First, we used the perturbation theory prediction for the response function of redshift-space power spectrum in the Fisher analysis, which breaks down in the deeply nonlinear regime. In order to realize the genuine functional form of the response function in the nonlinear regime, we need to use $N$-body simulations of large-scale structure formation and then study a coupling of the largescale tidal modes with small-scale Fourier modes. For doing this, a "separate universe simulation" method would be powerful, where the large-scale modes are absorbed into the background expansion. It was shown that this method works very well to model the response function to the large-scale density contrast, $\partial P(k) / \partial \delta_{\mathrm{b}}[21,22$, 28, 56, 57, 60, To extend this method, one can adopt an anisotropic expansion background to model the effect of the anisotropic super-survey mode, $\tau_{i j}$, and then run an $N$-body simulation onto the modified background [e.g., see Ref. 29 , for the related discussion]. If this separate universe simulation for $\tau_{i j}$ is developed, one can study various effects of $\tau_{i j}$ on nonlinear structures; the non-local bias of halos [48, 49], the correlation between $\tau_{i j}$ and shapes of halos [61, 62, and so on. This would be very interesting, and is our future work.

Another limitation of this paper is we used the redshift-space power spectrum, $P_{s}\left(k_{\perp}, k_{\|}\right)$, which is given as a function of two wavenumber variables such as $k_{\perp}$ and $k_{\|}$. Since the principle axes directions of $\tau_{i j}$ have nothing with the line-of-sight direction, the effect of $\tau_{i j}$ generally violates an statistical isotropy in the two-dimensional plane perpendicular to the line-of-sight direction. Hence, in order to fully extract the three-dimensional information on the tensor $\tau_{i j}$, one needs to use the redshift-space power spectrum given as a function of the three-dimensional vector, $P_{s}(\mathbf{k})$, without employing the angle average in the perpendicular plane as usually done in the standard method. Alternatively one can use a more general expansion of the redshift-space power spectrum, e.g. the bipolar spherical harmonics (BipoSH) decomposition [39]. It would be interesting to study how the full information on $\tau_{i j}$ can be 
extracted by using the BipSH decomposition.

Acknowledgments.- We thank Yin Li, Takahiro Nishimichi, Fabian Schmidt, Maresuke Shiraishi and Naonori Sugiyama for useful discussion, and we also thank to YITP, Kyoto University for their warm hospitality. KA is supported by the Advanced Leading Graduate Course for Photon Science at the University of Tokyo. MT is supported by World Premier International Research Center Initiative (WPI Initiative), MEXT, Japan, by the FIRST program "Subaru Measurements of Images and Redshifts (SuMIRe)", CSTP, Japan. MT is supported by Grant-in-Aid for Scientific Research from the JSPS Promotion of Science (No. 23340061, 26610058, and 15H05893), MEXT Grantin-Aid for Scientific Research on Innovative Areas (No. 15K21733, 15H05892) and by JSPS Program for Advancing Strategic International Networks to Accelerate the Circulation of Talented Researchers.

\section{Appendix A: Multipole power spectrum in the redshift-space power spectrum}

Here, we show the multipole expansion of $2 \mathrm{D}$ power spectrum in the redshift-space. The multipole power spectrum are defined as

$$
P_{s}^{\ell}\left(k ; \delta_{b}, \tau_{33}\right) \equiv(2 \ell+1) \int_{-1}^{1} \frac{d \mu}{2} P_{s W}^{2 D}\left(k, \mu ; \delta_{b}, \tau_{33}\right) \mathcal{L}_{\ell}(\mu)
$$

where $\mathcal{L}_{\ell}(\mu)$ is the Legendre polynomial. Making the use of Eq. (27) and Eq. (31), the multipole power spectra in the redshift-space can be calculated as

$$
\begin{aligned}
& P_{s}^{\ell=0}=\left[b^{2}+\frac{2}{3} b f+\frac{1}{5} f^{2}\right] P(k) \\
& +\delta_{b}\left[\left\{\frac{94}{21} b^{2}+\frac{52}{21} b f+2 b^{2} f+\frac{62}{105} f^{2}+\frac{4}{3} b f^{2}+\frac{26}{105} f^{3}\right\} P(k)\right. \\
& \left.-\left\{\frac{2}{3} b^{2}+2 b^{2} f+\frac{4}{9} b f+\frac{2}{9} b^{2} f+\frac{2}{15} f^{2}+\frac{4}{15} f b^{2}+\frac{2}{21} f^{3}\right\} \frac{\partial P(k)}{\partial \ln k}\right] \\
& +\tau_{33}\left[\left\{2 b^{2} f+\frac{32}{35} b f+\frac{128}{245} f^{2}+\frac{8}{5} b f^{2}+\frac{26}{35} f^{3}\right\} P(k)\right. \\
& \left.-\left\{\frac{8}{15} b f+\frac{2}{3} b^{2} f+\frac{8}{35} f^{2}+\frac{4}{5} b f^{2}+\frac{2}{7} f^{3}\right\} \frac{\partial P(k)}{\partial \ln k}\right] \\
& P_{s}^{\ell=2}=\left[\frac{4}{3} b f+\frac{4}{7} f^{2}\right] P(k) \\
& +\delta_{b}\left[\left\{\frac{8}{15} b^{2} f+\frac{104}{105} b f+\frac{16}{21} b f^{2}+\frac{248}{735} f^{2}+\frac{8}{45} f^{3}\right\} P(k)\right. \\
& \left.-\left\{\frac{4}{45} b^{2}+\frac{8}{45} b f+\frac{16}{105} b f^{2}+\frac{8}{105} f^{2}+\frac{4}{63} f^{3}\right\} \frac{\partial P(k)}{\partial \ln k}\right] \\
& +\tau_{33}\left[\left\{\frac{16}{35} b^{2}+\frac{176}{245} b f+\frac{96}{245} f^{2}+\frac{32}{35} b f^{2}+\frac{8}{15} f^{3}\right\} P(k)\right. \\
& \left.-\left\{\frac{2}{5} b^{2}+\frac{44}{105} b f+\frac{4}{15} b^{2} f+\frac{6}{35} f^{2}+\frac{16}{35} b f^{2}+\frac{4}{21} f^{3}\right\} \frac{\partial P(k)}{\partial \ln k}\right] \\
& P_{s}^{\ell=4}=\frac{8}{35} f^{2} P(k) \\
& +\delta_{b}\left[\left\{\frac{496}{6615} f^{2}+\frac{32}{189} b f^{2}+\frac{112}{1485} f^{3}\right\} P(k)\right. \\
& \left.-\left\{\frac{16}{945} f^{2}+\frac{32}{945} b f^{2}+\frac{16}{693} f^{3}\right\} \frac{\partial P(k)}{\partial \ln k}\right] \\
& +\tau_{33}\left[\left\{\frac{64}{245} b f+\frac{4352}{24255} f^{2}+\frac{64}{315} b f^{2}+\frac{112}{495} f^{3}\right\} P(k)\right. \\
& \left.-\left\{\frac{16}{105} b f+\frac{272}{3465} f^{2}+\frac{32}{315} b f^{2}+\frac{16}{231} f^{3}\right\} \frac{\partial P(k)}{\partial \ln k}\right]
\end{aligned}
$$




$$
\begin{aligned}
P_{s}^{\ell=6}= & \delta_{b}\left[\frac{128}{9009} f^{3} P(k)-\frac{32}{9009} f^{3} \frac{\partial P(k)}{\partial \ln k}\right] \\
& +\tau_{33}\left[\left\{\frac{256}{7007} f^{2}+\frac{128}{3003} f^{3}\right\} P(k)-\left\{\frac{16}{1001} f^{2}+\frac{32}{3003} f^{3}\right\} \frac{\partial P(k)}{\partial \ln k}\right]
\end{aligned}
$$

and the higher-multipole spectra with $\ell \geq 8$ vanish.

[1] M. Takada, E. Komatsu, and T. Futamase, Phys. Rev. D 73, 083520 (2006), arXiv:astro-ph/0512374

[2] M. Takada, R. S. Ellis, M. Chiba, J. E. Greene, H. Aihara, N. Arimoto, K. Bundy, J. Cohen, O. Doré, G. Graves, J. E. Gunn, T. Heckman, C. M. Hirata, P. Ho, J.-P. Kneib, O. L. Fèvre, L. Lin, S. More, H. Murayama, T. Nagao, M. Ouchi, M. Seiffert, J. D. Silverman, L. Sodré, D. N. Spergel, M. A. Strauss, H. Sugai, Y. Suto, H. Takami, and R. Wyse, PASJ 66, R1 (2014), arXiv:1206.0737.

[3] M. Takada and O. Doré, Phys. Rev. D 92, 123518 (2015), arXiv:1508.02469

[4] S. Alam, M. Ata, S. Bailey, F. Beutler, D. Bizyaev, J. A. Blazek, A. S. Bolton, J. R. Brownstein, A. Burden, C.-H. Chuang, J. Comparat, A. J. Cuesta, K. S. Dawson, D. J. Eisenstein, S. Escoffier, H. Gil-Marín, J. N. Grieb, N. Hand, S. Ho, K. Kinemuchi, D. Kirkby, F. Kitaura, E. Malanushenko, V. Malanushenko, C. Maraston, C. K. McBride, R. C. Nichol, M. D. Olmstead, D. Oravetz, N. Padmanabhan, N. Palanque-Delabrouille, K. Pan, M. Pellejero-Ibanez, W. J. Percival, P. Petitjean, F. Prada, A. M. Price-Whelan, B. A. Reid, S. A. Rodríguez-Torres, N. A. Roe, A. J. Ross, N. P. Ross, G. Rossi, J. A. Rubiño-Martín, S. Saito, S. Salazar-Albornoz, L. Samushia, A. G. Sánchez, S. Satpathy, D. J. Schlegel, D. P. Schneider, C. G. Scóccola, H.-J. Seo, E. S. Sheldon, A. Simmons, A. Slosar, M. A. Strauss, M. E. C. Swanson, D. Thomas, J. L. Tinker, R. Tojeiro, M. V. Magaña, J. A. Vazquez, L. Verde, D. A. Wake, Y. Wang, D. H. Weinberg, M. White, W. M. Wood-Vasey, C. Yèche, I. Zehavi, Z. Zhai, and G.-B. Zhao, Mon. Not. Roy. Astron. Soc. 470, 2617 (2017), arXiv:1607.03155.

[5] S. Dodelson, Modern cosmology / Scott Dodelson. Amsterdam (Netherlands): Academic Press. ISBN 0-12-219141-2, 2003, $X I I I+440$ p. (2003).

[6] Planck Collaboration, P. A. R. Ade, N. Aghanim, M. Arnaud, M. Ashdown, J. Aumont, C. Baccigalupi, A. J. Banday, R. B. Barreiro, J. G. Bartlett, and et al., Astronomy \& Astrophysics 594, A13 (2016), arXiv:1502.01589.

[7] F. Bernardeau, S. Colombi, E. Gaztañaga, and R. Scoccimarro, Phys. Rep. 367, 1 (2002), arXiv:astro-ph/0112551

[8] V. Desjacques, D. Jeong, and F. Schmidt, ArXiv e-prints (2016), arXiv:1611.09787

[9] M. Takada and B. Jain, Mon. Not. Roy. Astron. Soc. 340, 580 (2003), arXiv:astro-ph/0209167

[10] M. Takada and W. Hu, Phys. Rev. D 87, 123504 (2013), arXiv:1302.6994 [astro-ph.CO].

[11] A. J. S. Hamilton, C. D. Rimes, and R. Scoccimarro, Mon. Not. Roy. Astron. Soc. 371, 1188 (2006), arXiv:astro$\mathrm{ph} / 0511416$

[12] E. Sefusatti, M. Crocce, S. Pueblas, and R. Scoccimarro, Phys. Rev. D 74, 023522 (2006), arXiv:astro-ph/0604505

[13] M. Takada and S. Bridle, New Journal of Physics 9, 446 (2007), arXiv:arXiv:0705.0163

[14] M. Takada and B. Jain, Mon. Not. Roy. Astron. Soc. 395, 2065 (2009), arXiv:0810.4170

[15] M. Sato, T. Hamana, R. Takahashi, M. Takada, N. Yoshida, T. Matsubara, and N. Sugiyama, Astrophys. J. 701, 945 (2009), arXiv:0906.2237 [astro-ph.CO].

[16] T. Baldauf, U. Seljak, L. Senatore, and M. Zaldarriaga, JCAP 10, 031 (2011), arXiv:1106.5507.

[17] B. D. Sherwin and M. Zaldarriaga, Phys. Rev. D 85, 103523 (2012), arXiv:1202.3998 [astro-ph.CO].

[18] R. de Putter, C. Wagner, O. Mena, L. Verde, and W. J. Percival, JCAP 4, 019 (2012), arXiv:1111.6596.

[19] M. Takada and D. N. Spergel, ArXiv e-prints (2013), arXiv:1307.4399 [astro-ph.CO]

[20] E. Schaan, M. Takada, and D. N. Spergel, ArXiv e-prints (2014), arXiv:1406.3330.

[21] Y. Li, W. Hu, and M. Takada, Phys. Rev. D 89, 083519 (2014), arXiv:1401.0385.

[22] Y. Li, W. Hu, and M. Takada, Phys. Rev. D 90, 103530 (2014), arXiv:1408.1081.

[23] R. Takahashi, S. Soma, M. Takada, and I. Kayo, Mon. Not. Roy. Astron. Soc. 444, 3473 (2014), arXiv:1405.2666

[24] A. Manzotti, W. Hu, and A. Benoit-Lévy, Phys. Rev. D 90, 023003 (2014), arXiv:1401.7992

[25] J. Carron and I. Szapudi, Mon. Not. Roy. Astron. Soc. 447, 671 (2015), arXiv:1408.1744.

[26] L. Dai, E. Pajer, and F. Schmidt, JCAP 10, 059 (2015), arXiv:1504.00351

[27] M. Shirasaki, M. Takada, H. Miyatake, R. Takahashi, T. Hamana, T. Nishimichi, and R. Murata, Mon. Not. Roy. Astron. Soc. 470, 3476 (2017), arXiv:1607.08679.

[28] Y. Li, W. Hu, and M. Takada, Phys. Rev. D 93, 063507 (2016) arXiv:1511.01454.

[29] H. Y. Ip and F. Schmidt, JCAP 2, 025 (2017), arXiv:1610.01059.

[30] K. Akitsu, M. Takada, and Y. Li, Phys. Rev. D 95, 083522 (2017), arXiv:1611.04723.

[31] A. Barreira and F. Schmidt, ArXiv e-prints (2017), arXiv:1703.09212

[32] E. Krause, T. F. Eifler, J. Zuntz, O. Friedrich, M. A. Troxel, S. Dodelson, J. Blazek, L. F. Secco, N. MacCrann, E. Baxter, C. Chang, N. Chen, M. Crocce, J. DeRose, A. Ferte, N. Kokron, F. Lacasa, V. Miranda, Y. Omori, A. Porredon, R. Rosenfeld, S. Samuroff, M. Wang, R. H. Wechsler, T. M. C. Abbott, F. B. Abdalla, S. Allam, J. Annis, K. Bechtol, A. Benoit-Levy, G. M. Bernstein, D. Brooks, D. L. Burke, D. Capozzi, M. Carrasco Kind, J. Carretero, C. B. D'Andrea, L. N. da Costa, C. Davis, D. L. DePoy, S. Desai, H. T. Diehl, J. P. Dietrich, A. E. Evrard, B. Flaugher, P. Fosalba, 
J. Frieman, J. Garcia-Bellido, E. Gaztanaga, T. Giannantonio, D. Gruen, R. A. Gruendl, J. Gschwend, G. Gutierrez, K. Honscheid, D. J. James, T. Jeltema, K. Kuehn, S. Kuhlmann, O. Lahav, M. Lima, M. A. G. Maia, M. March, J. L. Marshall, P. Martini, F. Menanteau, R. Miquel, R. C. Nichol, A. A. Plazas, A. K. Romer, E. S. Rykoff, E. Sanchez, V. Scarpine, R. Schindler, M. Schubnell, I. Sevilla-Noarbe, M. Smith, M. Soares-Santos, F. Sobreira, E. Suchyta, M. E. C. Swanson, G. Tarle, D. L. Tucker, V. Vikram, A. R. Walker, and J. Weller, ArXiv e-prints (2017), arXiv:1706.09359

[33] F. Schmidt, E. Pajer, and M. Zaldarriaga, Phys. Rev. D 89, 083507 (2014), arXiv:1312.5616

[34] N. Kaiser, Mon. Not. Roy. Astron. Soc. 227, 1 (1987).

[35] A. J. S. Hamilton, in The Evolving Universe, Astrophysics and Space Science Library, Vol. 231, edited by D. Hamilton (1998) pp. 185-+.

[36] C. Alcock and B. Paczynski, Nature (London) 281, 358 (1979).

[37] H. Seo and D. J. Eisenstein, Astrophys. J. 598, 720 (2003), arXiv:astro-ph/0307460

[38] W. Hu and Z. Haiman, Phys. Rev. D 68, 063004 (2003), astro-ph/0306053

[39] M. Shiraishi, N. S. Sugiyama, and T. Okumura, Phys. Rev. D 95, 063508 (2017), arXiv:1612.02645.

[40] M. H. Goroff, B. Grinstein, S.-J. Rey, and M. B. Wise, Astrophys. J. 311, 6 (1986)

[41] N. Makino, M. Sasaki, and Y. Suto, Phys. Rev. D 46, 585 (1992).

[42] B. Jain and E. Bertschinger, Astrophys. J. 431, 495 (1994), astro-ph/9311070.

[43] E. Hivon, F. R. Bouchet, S. Colombi, and R. Juszkiewicz, Astronomy \& Astrophysics 298, 643 (1995), astro-ph/9407049

[44] L. Verde, A. F. Heavens, S. Matarrese, and L. Moscardini, Mon. Not. Roy. Astron. Soc. 300, 747 (1998), astro-ph/9806028

[45] R. Scoccimarro, H. M. P. Couchman, and J. A. Frieman, Astrophys. J. 517, 531 (1999), astro-ph/9808305.

[46] R. Scoccimarro, Phys. Rev. D 70, 083007 (2004), arXiv:astro-ph/0407214.

[47] P. McDonald and A. Roy, JCAP 8, 020 (2009), arXiv:0902.0991 [astro-ph.CO].

[48] K. C. Chan, R. Scoccimarro, and R. K. Sheth, Phys. Rev. D 85, 083509 (2012), arXiv:1201.3614 [astro-ph.CO].

[49] S. Saito, T. Baldauf, Z. Vlah, U. Seljak, T. Okumura, and P. McDonald, Phys. Rev. D 90, 123522 (2014) arXiv:1405.1447

[50] C.-T. Chiang, C. Wagner, F. Schmidt, and E. Komatsu, ArXiv e-prints (2014), arXiv:1403.3411 [astro-ph.CO].

[51] T. Nishimichi and P. Valageas, Phys. Rev. D 92, 123510 (2015), arXiv:1503.06036.

[52] H.-J. Seo and D. J. Eisenstein, Astrophys. J. 665, 14 (2007), astro-ph/0701079

[53] D. J. Eisenstein, H.-J. Seo, E. Sirko, and D. N. Spergel, Astrophys. J. 664, 675 (2007), arXiv:astro-ph/0604362.

[54] E. Komatsu, K. M. Smith, J. Dunkley, C. L. Bennett, B. Gold, G. Hinshaw, N. Jarosik, D. Larson, M. R. Nolta, L. Page, D. N. Spergel, M. Halpern, R. S. Hill, A. Kogut, M. Limon, S. S. Meyer, N. Odegard, G. S. Tucker, J. L. Weiland, E. Wollack, and E. L. Wright, Astrophys. J. Suppl. 192, 18 (2011), arXiv:1001.4538 [astro-ph.CO]|

[55] N. Padmanabhan, X. Xu, D. J. Eisenstein, R. Scalzo, A. J. Cuesta, K. T. Mehta, and E. Kazin, Mon. Not. Roy. Astron. Soc. 427, 2132 (2012), arXiv:1202.0090

[56] C. Wagner, F. Schmidt, C.-T. Chiang, and E. Komatsu, Mon. Not. Roy. Astron. Soc. 448, L11 (2015), arXiv:1409.6294

[57] T. Baldauf, U. Seljak, L. Senatore, and M. Zaldarriaga, JCAP 9, 007 (2016), arXiv:1511.01465.

[58] D. Jeong and M. Kamionkowski, Physical Review Letters 108, 251301 (2012), arXiv:1203.0302.

[59] C. Byrnes, G. Domènech, M. Sasaki, and T. Takahashi, JCAP 12, 020 (2016), arXiv:1610.02650.

[60] W. Hu, C.-T. Chiang, Y. Li, and M. LoVerde, Phys. Rev. D 94, 023002 (2016), arXiv:1605.01412.

[61] N. E. Chisari, C. Dvorkin, F. Schmidt, and D. N. Spergel, Phys. Rev. D 94, 123507 (2016), arXiv:1607.05232

[62] T. Okumura, T. Nishimichi, K. Umetsu, and K. Osato, ArXiv e-prints (2017), arXiv:1706.08860. 